\title{
Transmission Lines to Enhance the Reading of UHF RFID Tags on Small Metallic Objects
}

\author{
Imbolatiana Rakotomalala \\ Univ. Grenoble Alpes \\ Grenoble INP, LCIS \\ Valence, France \\ imbolatiana.rakotomalala@lcis.grenoble-inp.fr
}

\author{
Pierre Lemaitre Auger \\ Univ.Grenoble Alpes \\ Grenoble INP, LCIS \\ Valence, France
}

pierre.lemaitre-auger@1cis.grenoble-inp.fr

\author{
Smaïl Tedjini \\ Univ. Grenoble Alpes \\ Grenoble INP, LCIS \\ Valence, France \\ smail.tedjini@1cis.grenoble-inp.fr
}

\begin{abstract}
This paper presents an antenna-like passive structure that allows to read miniaturized UHF RFID tags attached to small metallic objects. The solution is realized by combing far-field Wireless Power Transfer (WPT) with a Conductor-Baked Coplanar Waveguide (CBCPW) transmission line. Low cost proof-of-concept prototypes were realized on a FR4 substrate. Measurements are in good agreement with simulations. A maximum read range of $2.20 \mathrm{~m}$ can be achieved for the far-field operation.
\end{abstract}

Keywords - Passive enhancement structure, CBCPW transmission line, Miniature RFID tag, Near-field UHF RFID

\section{INTRODUCTION}

Nowadays, automatic identification data collection systems have become very useful and popular in many applications. Thanks to the advantages of large amount of stored data, fast reading speed, and no need of human intervention to read the data, Radio Frequency Identification (RFID) has been widely adopted [1]

The management of small precious assets with RFID technologies is an interesting challenge, especially when those assets are small metallic items. Recently, Ultra-High Frequency (UHF) near-field RFID technology received a lot of attention due to the promising opportunities in item-level RFID applications such as sensitive products tracking, pharmaceutical, logistics, transport and medical products [2]. Moreover, near-field systems allow a considerable size reduction of tags compare to those used in far-field systems, expending the range of applications of RFID to extremely small items [3].

In many existing applications of the near-field UHF RFID, the reader is directly attached to the antenna that generates a strong enough and well distributed magnetic field to ensure the best reading rate in the interrogation area. The tagged objects (TOs) are placed optimally in the interrogation area. As the communication is performed in a near field region, hence, the reading distance is in the order of few centimeters [4]-[8]. However, these types of solutions are not practical for an operation in the far field, since the free space loss will weaken the field generate by the antenna in near-field.

In this work, we propose and study the performance of an antenna-like structure capable of reading several miniaturized UHF RFID tags mounted on small metallic objects. The proposed solution is fully passive, low cost and can produce strong and uniform magnetic field in the region near to the antenna, even for a far field operation. It is a CBCPW transmission line [7], and it can easily be inserted in foam supports which also comprise the space for the positioning of the TOs. That way, the relative placement of the TOs to the antenna-like structure is ensured. The proposed solution is tolerant to the angular orientation of the objects within their place.

This paper is organized as follows. In section II, the characteristics of the considered solution is presented, the theoretical model and analysis are discussed. In section III, experimental validation of the proposed solution is presented.

\section{General CONFIGURATION}

The Fig. 1 shows the schematic diagram of the studied configuration. The solution is composed of a CBCPW transmission line terminated by a matching load, i.e. $50 \mathrm{Ohm}$ in the present case. This line is connected to the RFID reader either directly by a coaxial cable, either through a relay antenna system [9] if the reader is not located in the same space as the tags to be read. With this configuration TOs are arranged optimally with respect to CBCPW line, i.e. the axes of the TOs is parallel to the transmission line plane and perpendicular to the direction of the transmission line.

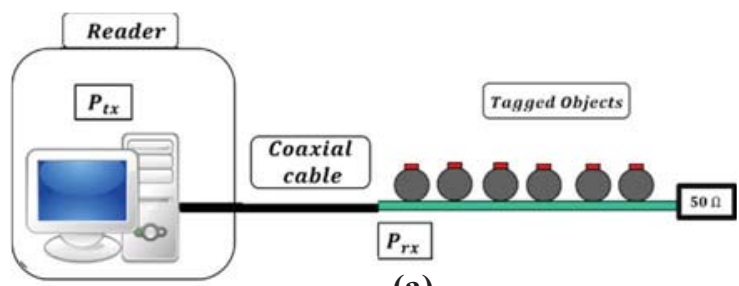

(a)

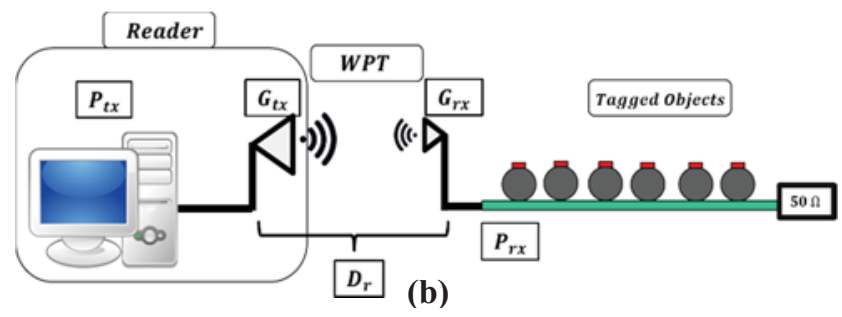

Fig. 1. Schematic representation of the antenna-like structure: (a) direct connection; (b) with WPT for far field operation.

Fig. 2 shows the geometry of the CBCPW. It consists of three coplanar metal strips of finite width, $w$ and $g$ for the center and side strips, respectively. The distance between two neighborin strips is denoted by "s. For this study the structural parameters and the material constants are so chosen that the structure supports only the CPW mode and the MicrostripLike mode below $1 \mathrm{GHz}$. The overall dimensions of the CBCPW line are $200 \times 20 \mathrm{~mm}$. A $1.6 \mathrm{~mm}$ FR4 substrate is used. The central line width " $\mathrm{w}$ " is $1.8 \mathrm{~mm}$, and the side strips width " $\mathrm{g}$ " is while the distance between two neighborin strips "s" is $1.5 \mathrm{~mm}$ so that a $\sim 50 \Omega$ impedance line is obtained [10]. In its immediate surroundings, it creates and uniform magnetic field strong enough to awake the RFID chip. 


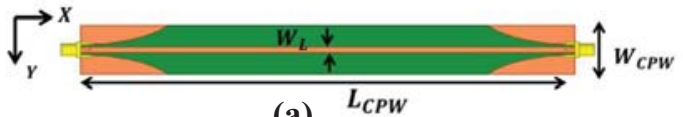

(a)

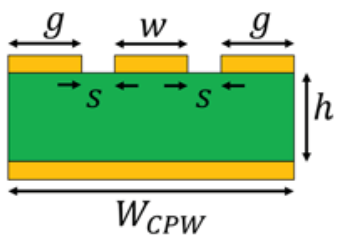

(b)

Fig. 2 Top view of the CBCPW line configuration.

To detect tags, it is necessary that the power received at the terminals of the tag antenna, $P_{T a g}$, is higher than the minimum power needed to activate the chip, $P_{t h}[11]$. For near field UHF RFID system, this power is:

$$
P_{\text {Tag }}=P_{\text {Reader }} \rho C_{N f} \tau
$$

where $P_{\text {reader }}$ is the power emitted by the RFID reader, $\rho$ is the impedance matching coefficient between the reader and its antenna, $C_{N f}$ the coupling coefficient between the CBCPW line and the RFID tag, and $\tau$ is the impedance matching coefficient between the tag chip and its antenna.

However, when the tag is located in the far field region, the coupling coefficient is different and can be written as:

$$
C_{F f}=G_{t x} L_{p a t h} G_{r x} p
$$

where $G_{t x}$ is the gain of the transmitting reader antenna, $L_{p a t h}$ is the propagation path loss, $G_{r x}$ is the gain of the receiving relay antenna, and $p$ is the polarization mismatch loss between the reader antenna and relay antenna. In free space, the path loss is given by well known Friis equation:

$$
L_{\text {path }}=\left(\frac{\lambda}{4 \pi d}\right)^{2}
$$

where $\mathrm{d}$ is the distance between the reader antenna and relay antenna.

Combining (1), (2), and (3), we can obtain the total power received $P_{\text {TagTot }}$, at the terminals of the tag antenna for the configuration depicted in Fig. 1 (b) as :

$$
P_{\text {TagTot }}=P_{\text {Reader }} \rho C_{N f} \tau G_{t x} G_{r x} p\left(\frac{\lambda}{4 \pi d}\right)^{2}
$$

In the present case, considering that the TO are small enough not to disturb the magnetic field created by the CBCPW line, the coupling coefficient $C_{N f}$ depends on the frequency of operation, the distance between the central line and the tag, the missalignement between the tags and the line Hence, according to [3][12],the magnitude of the magnetic field normal to the surface of the tag (referred to as $\left|H_{z}\right|$ ) should be greater than the threshold value $H_{t h}$ which is about $-30 \mathrm{dBA} / \mathrm{m}$ for the Dash XXS by Xeraf, a passive UHF Class 1 Gen 2 tag [13]. It is equipped with the Alien Higgs-3 chip that has a typical sensitivity, $P_{t h}$, of $-17 \mathrm{dBm}$.

Full wave simulations were done using Ansys HFSS and the simulated magnetic fields are evaluate at $868 \mathrm{MHz}$. The top view and the side view of the simulated magnetic field distribution at are shown in Fig. 3. Top view is $1 \mathrm{~mm}$ above the transmission line while the side view is at the center of it.

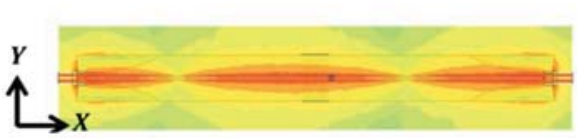

(a)

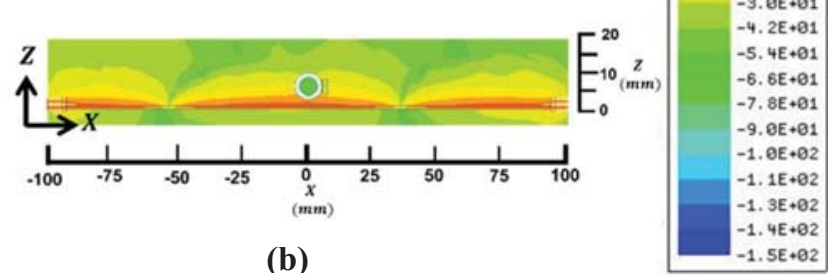

Fig. 3. Simulated distribution of $\left|H_{z}\right|$ at the time $t=0$. (a) At an a height of $1 \mathrm{~mm}$ above the transmission line. (b) Side view centered on the transmission line $(y=0)$.

Some minimum values can be seen in Fig. 3. This is naturally caused by the traveling nature of the electromagnetic field in the transmission line. The length between two zeros is: $100 \mathrm{~mm}$, which correspond to $\sim \lambda_{\text {eff }} / 2$ at $f=868 \mathrm{MHz}, \varepsilon_{\text {eff }}$ is indeed equal to 3.031 in the transmission line. In Fig $4, H_{z}$ is computed along the length of transmission line, at the center of it, and for different time. It can be seen that the maximum value of $H_{z}$ is almost constant all along the transmission line length.

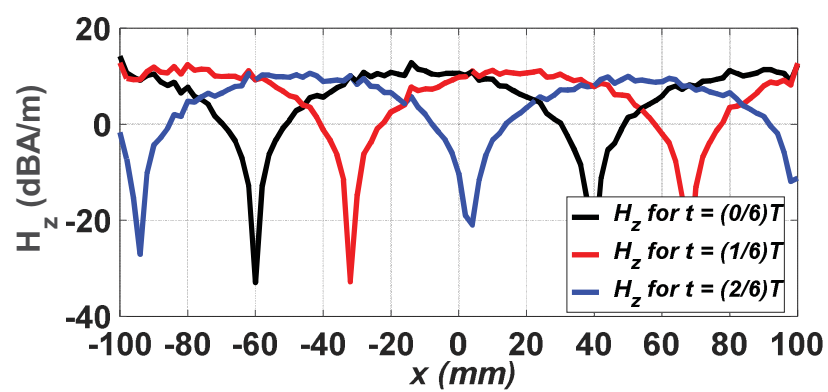

Fig. 4. Simulated $\left|H_{z}\right|$ value of the proposed line for different period

The simulated values of the magnetic field at several observing height ( 1 to $20 \mathrm{~mm}$ ) over the transmission line and $y=0$ for an input power of $15.24 \mathrm{dBm}$ at the entrance of the transmission line is shown in Fig. 5.

When the observing height is below $10 \mathrm{~mm}$, strong values of $\left|H_{z}\right|$ greater to $-20 \mathrm{dBA} / \mathrm{m}$ are achieved within the tag's antenna area for all $x$ positions. At the height of $15 \mathrm{~mm}$, the limit value of $-30 \mathrm{dBA} / \mathrm{m}$ is reached.

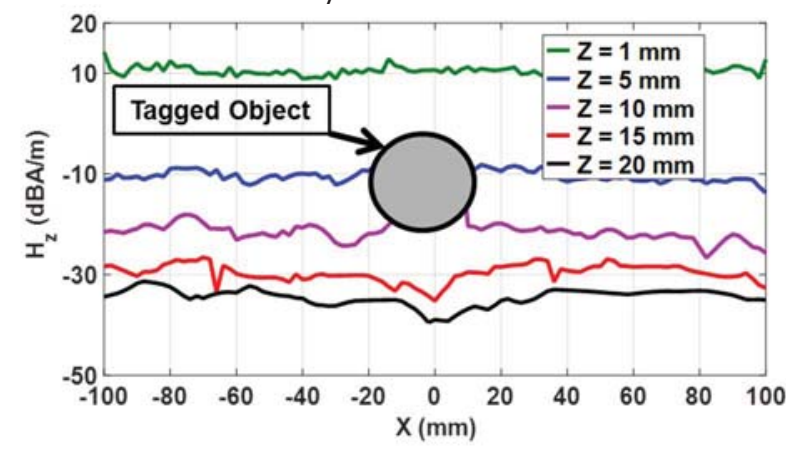

Fig. 5. Simulated $\left|H_{z}\right|$ value with different observing height.

Theses first simulated results show that a single transmission line is very well adapted to detect a miniature metallic object with a tag height below $15 \mathrm{~mm}$. This configuration is thus suitable for applications where the user wants to track TO that can be placed at a fixed location. 
Precise position can indeed easily be obtained with preformed foams.

However, for objects having rotational symmetry, the angular orientation can't usually be controlled, resulting in an uncertainty on the tag's height. In the latter case, the magnetic field is also evaluated for all angular positions of the TO $(0$ to $360^{\circ}$ ) at $868 \mathrm{MHz}$. In this simulation a small metallic tool (a $7 \mathrm{~mm}$ socket, diameter of $11.2 \mathrm{~mm}$ ) is used. As expected, a maximum value of $16 \mathrm{dBA} / \mathrm{m}$ is observed at $0^{\circ}$ while a minimum value $-28 \mathrm{dBA} / \mathrm{m}$ is observed at $180^{\circ}$ as depicted in Fig. 6. Hence, we can consider that the maximum diameter of a symmetrical tool should be $15 \mathrm{~mm}$ in order to be detected by the structure

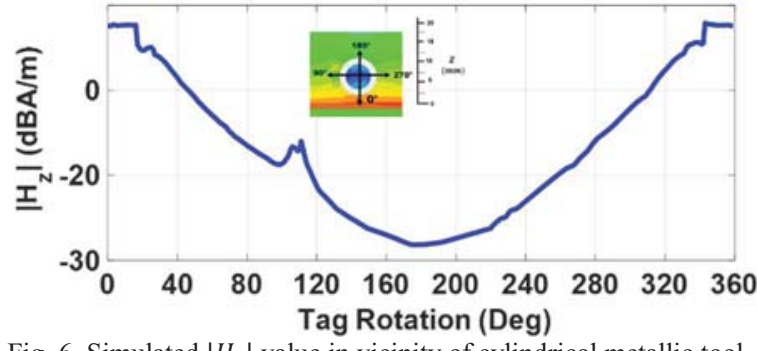

Fig. 6. Simulated $\left|H_{z}\right|$ value in vicinity of cylindrical metallic tool

\section{EXPERIMENTAL VALIDATION}

In order to validate the solution, a prototype of the proposed $\mathrm{CBCPW}$ line has been fabricated. The reflection coefficient of the antenna-like structure is measured and compared to the simulated one. It shows a broadband behavior, which is from 800 to $1000 \mathrm{MHz}$ (with $\left|S_{11}\right|<-10$ ).

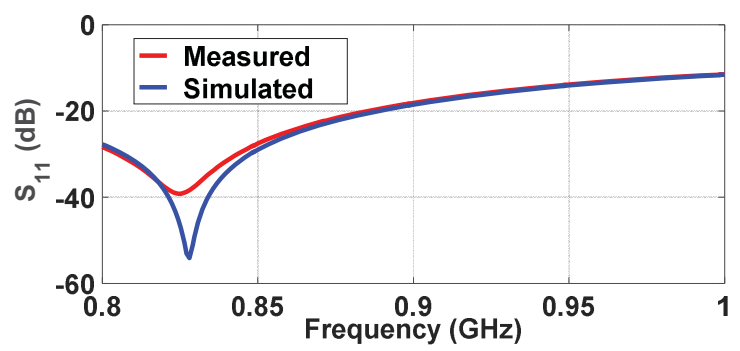

Fig. 7. Simulated and measured reflection coefficient of the line

Fig. 8 shows the test environment used to evaluate the far field reading performance of the solution. The relay antenna with gain of $1 \mathrm{dBi}$, is placed $30 \mathrm{~cm}$ away from the reader antenna, measurement is done with Tagformance Voyantic platform [14]. A $7 \mathrm{~mm}$ sockets is used for the measurement, where the tags is attached directly on the surface of the metallic tool.

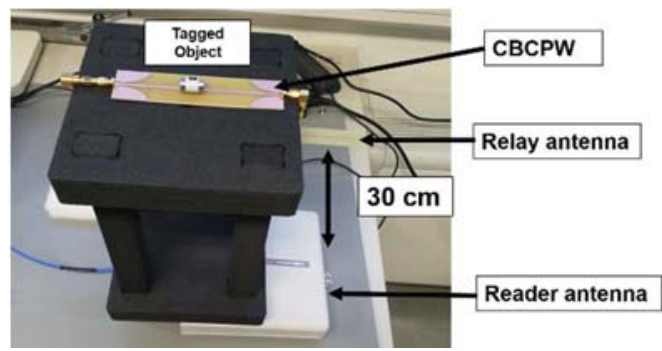

Fig. 8. Measurement experiment setup.
We evaluate the read range of the tag, for 3 different orientation, and as depicted in Fig. 9 maximum read range is observed at $0^{\circ}(2.20 \mathrm{~m})$, while the minimal read range is observed at $180^{\circ}(1.22 \mathrm{~m})$, as expected where the magnetic field distributions are less intense.

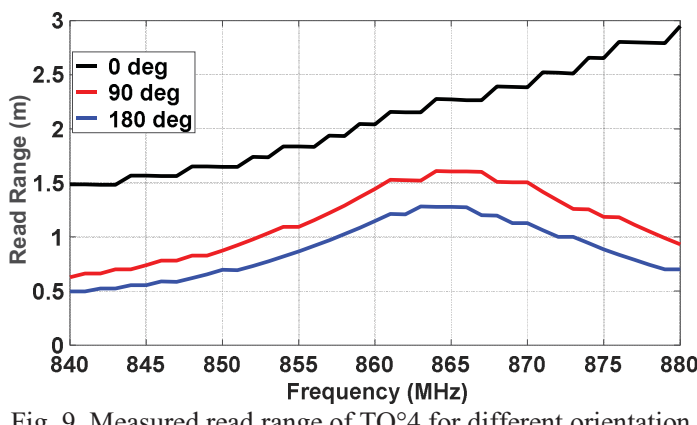

\section{CONCLUSION}

An antenna-like structure, a CBCPW line, for the reading of small metallic tagged objects is presented with UHF RFID readers. A low cost prototype of the solution was fabricated on FR4 and tested. Results demonstrate a read range of 2.20 $\mathrm{m}$ can be achieved in the best case while $1.22 \mathrm{~m}$ for the worst case. More results will be presented at the conference.

\section{REF ERENCES}

[1] K. Finkenzeller, RFID Handbook. Chichester, UK: John Wiley \& Sons, Ltd., 2003.

[2] T. For, D. Transformation, A. F. O. R. Efficiency, T. For, and D. Transformation, "Industry solutions." [Online]. Available: https://www.impinj.com/solutions/.

[3] L. Shen, C. Huang, C. Wang, W. Tang, W. Zhuang, and J. Xu, "A Yagi - Uda Antenna With Load and Additional Reflector for Near-Field UHF RFID," vol. 16, pp. 728-731, 2017.

[4] X. Qing, C. K. Goh, and Z. N. Chen, "A Broadband UHF NearField RFID Antenna,” IEEE Trans. Antennas Propag., vol. 58, no. 12 , pp. 3829-3838, 2010.

[5] U. H. F. N. Rfid and R. Antenna, "Electrically Large Zero-PhaseShift Line Grid-Array," IEEE Trans. Antennas Propag., vol. 62, no. 2, pp. 2201-2208, 2014

[6] X. Gu and W. Geyi, "Design of a Near-Field RFID Antenna Array in," IEEE Antennas Wirel. Propag. Lett., vol. 18, no. 1, pp. 79-83, 2019.

[7] C. R. Medeiros, J. R. Costa, and C. A. Fernandes, "RFID smart shelf with confined detection volume at UHF," IEEE Antennas Wirel. Propag. Lett., vol. 7, pp. 773-776, 2008.

[8] C. Wu, J. Du, A. Ren, and J. Li, "Large-area RFID reader antenna for smart shelf at UHF," vol. 48, no. 15, pp. 1-2, 2012.

[9] I. Rakotomalala and S. Tedjini, "UHF Near-Field Wireless Power Transfer, Application To RFID System,” no. June, p. 2018, 2018.

[10] D. Pozar, Microwave Engineering Fourth Edition. 2005.

[11] M. Daiki, "Contribution au développement d' antennes lecteurs champ proche pour les systèmes RFID UHF passifs To cite this version : HAL Id : tel-01179964 Contribution au développement d' antennes lecteur champ proche pour les systèmes RFID UHF passifs," 2015.

[12] "Higgs-3." [Online]. Available: www.alientechnology.com.

[13] "Dash XXS." [Online]. Available: www.xerafy.com.

[14] "Tagformance pro." [Online]. Available: http://voyantic.com/. 